\title{
Outpatient Care of COVID-19 Patients
}

\section{V.4.0/12.01.2021; provided by med/X}

1. Patient tests positive for COVID-19

2. General practitioner (GP) identifies additional risk factors:
- Diabetes
- Renal/liver disease
- Pregnancy
Immunosuppression
- COPD/asthma/smoking
- Cardiovascular disease

- Obesity

3. GP discusses with patient:

- Hospitalization

- Resuscitation, intensive care/intubation

- Advance directive/Emergency care order/Advance Care Planning (ACP)

4. Poor clinical general condition or deterioration of general condition during course (self or relative monitoring)

5. Is patient home alone or is hospitalization required for other reasons?

6. Delivery of a pulse oximeter ${ }^{1}$ and instruction of the patient regarding:

- Correct self-measurement of respiratory rate and saturation 4 times a day

- Measuring frequency of pulse, blood pressure, temperature (depending on comorbidity)

- Drinking 6-8 glasses of fluid a day

In case of a relevant thrombotic event in the past due

to being bedridden or active tumor disease:

consider thrombosis prophylaxis ${ }^{2}$.

GP defines with patient feedback interval and mode of communication:

- GP $24 \mathrm{~h}$

Palliative or COVID

- Advanced Nurse Practitioner/ Medical Practice Coordinators

home care

- Nursing Home

- GP daytime/nighttime emergency physician or telemedicine

7. Measurements according to instruction \& regular feedback:
- Vital signs
- Little urine
- Cyanosis
- Meningism
- Speech dyspnea
- Hemoptysis
- Dyspnea
- Sustained rash

- Chest pressure

If improvement is sustained, discontinue monitoring. If worsening, especially if:

- Newly confused

- Saturation $<92 \%$

- Respiratory rate $>22 / \mathrm{min}$

- Pulse over 100/min

8. Adjust treatment setting: Decide with patient about hospitalization or home care.

9. Organize home care setting:

- Prescribe oxygen: only if hospitalization is not desired (prescription 4 Lungenliga)

- Organize palliative or COVID home care, if necessary

- Palliative prescription

- Consider dexamethasone ${ }^{3}$

10. Organize hospitalization

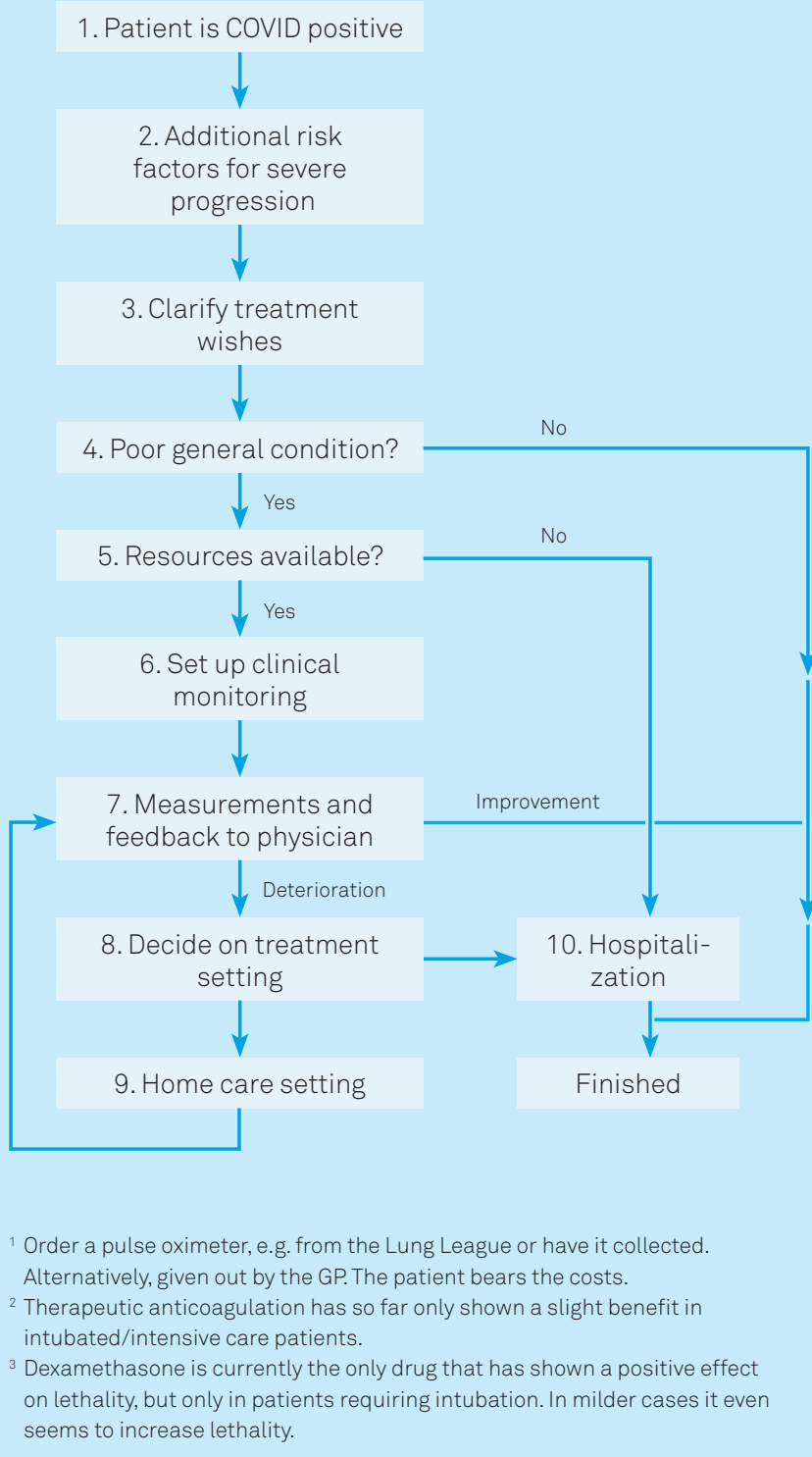

PD Dr. med. Corinne Chmiel

mediX switzerland

corinne.chmiel@medix.ch

This fact sheet was prepared with no financial or content-related influence by industry or other institutions or interest groups. It contains therapeutic recommendations for specific symptoms or treatment situations. However, each patient must be treated according to his or her individual circumstances. This factsheet has been developed and checked with great care, but the mediX association cannot guarantee its accuracy. 